\title{
Measurements of Collins and Sivers asymmetries at COMPASS
}

Paolo Pagano* a on behalf of the COMPASS collaboration

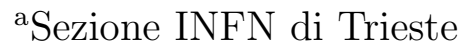

Padriciano 99, 34012 Padriciano (TS), Italy

E-mail: Paolo.Pagano@cern.ch

COMPASS is a fixed target experiment presently running at CERN. In 2002, 2003, and 2004 the experiment used a $160 \mathrm{GeV}$ polarized muon beam coming from SPS and scattered off a ${ }^{6} \mathrm{LiD}$ target. The nucleons in the target can be polarized either longitudinally or transversely with respect to the muon beam and $20 \%$ of the running time has been devoted to transverse polarization. From the transverse polarization data collected in 2002, which correspond to a total integrated luminosity of about $200 \mathrm{pb}^{-1}$, the Collins and the Sivers asymmetries have been determined separately and the preliminary results are presented here.

\section{The theoretical framework}

The cross-section for polarized deep inelastic scattering[ [1] of leptons off spin $1 / 2$ hadrons can be expressed, at the leading twist, as a function of three independent quark distribution function: $q(x), \Delta q(x)$ and $\Delta_{T} q(x)$. The latter is chiral-odd and can be measured in combination with a chiral-odd fragmentation function, the Collins function $\Delta D_{a}^{h}\left(z, p_{T}^{h}\right)$, via azimuthal single spin asymmetries [2] (SSA) in the hadronic end-product (semi-inclusive measurement). A similar effect can arise from a possible quark $k_{T}$ structure of a transversely polarized nucleon (the Sivers function, $\Delta_{0}^{T} q$ ), which also causes an azimuthal asymmetry in the produced hadrons. Leptoproduction on transversely polarized nucleons is a favourable setting to disentangle the Collins and Sivers effects since they show a dependence from linearly independent kinematic variables.

According to Collins, the fragmentation function of a quark of flavour $a$ in a hadron $h$ can be written as[ [3]:

$$
D_{a}^{h}\left(z, \mathbf{p}_{\mathbf{T}}^{\mathbf{h}}\right)=D_{a}^{h}\left(z, p_{T}^{h}\right)+\Delta D_{a}^{h}\left(z, p_{T}^{h}\right) \cdot \sin \Phi_{C}
$$

where $\mathbf{p}_{\mathbf{T}}^{\mathbf{h}}$ is the final hadron transverse momentum with respect to the quark direction i.e. the virtual photon direction - and $z=E_{h} /\left(E_{l}-E_{l^{\prime}}\right)$ is the fraction of available energy carried by the hadron ( $E_{h}$ is the hadron energy, $E_{l}$ is the incoming lepton energy and $E_{l^{\prime}}$ is the scattered lepton energy). The angle appearing in the fragmentation function, known as "Collins angle" and noted as $\Phi_{C}$, is conveniently defined in the system where the z-axis

${ }^{*}$ This paper will be published in the proceedings of the " $16^{\text {th }}$ International Spin Physics Symphosium SPIN 2004", October 10-16, 2004, Trieste, Italy. 
is the virtual photon direction and the $\mathrm{x}-\mathrm{z}$ plane is the muon scattering plane. In this frame $\Phi_{C}=\Phi_{h}-\Phi_{s}^{\prime}$, where $\Phi_{h}$ is the hadron azimuthal angle, and $\Phi_{s}^{\prime}$ is the azimuthal angle of the transverse spin of the struck quark. Since $\Phi_{s}^{\prime}=\pi-\Phi_{s}$, with $\Phi_{s}$ the azimuthal angle of the transverse spin of the initial quark (nucleon), the relation $\Phi_{C}=\Phi_{h}+\Phi_{s}-\pi$ is also valid. The fragmentation function $\Delta D_{a}^{h}\left(z, p_{T}^{h}\right)$ couples to transverse spin distribution function $\Delta_{T} q(x)$ and gives rise to SSA (denoted as $A_{C o l l}$ ) dependent on $x, z$ and $p_{T}^{h}$ kinematic variables.

Following the Sivers hypothesis, the difference in the probability of finding an unpolarised quark of transverse momentum $\mathbf{k}_{\mathbf{T}}$ and $-\mathbf{k}_{\mathbf{T}}$ inside a polarised nucleon can be written as [6]:

$$
\mathrm{P}_{q / p^{\uparrow}}\left(x, \mathbf{k}_{\mathbf{T}}\right)-\mathrm{P}_{q / p^{\uparrow}}\left(x,-\mathbf{k}_{\mathbf{T}}\right)=\sin \Phi_{S} \Delta_{0}^{T} q\left(x, k_{T}^{2}\right)
$$

where $\Phi_{S}=\Phi_{k}-\Phi_{s}$ is the azimuthal angle of the quark with respect to the nucleon transverse spin orientation. It has been recently demonstrated by theoretical arguments [ 17, 8], that SSA (denoted as $A_{\text {Siv }}$ ) coming from the coupling of the Sivers function with the un-polarised fragmentation function $D_{a}^{h}\left(z, p_{T}^{h}\right)$ can be observed at the leading twist from polarised Semi-Inclusive DIS.

\section{The COMPASS 2002 run for transversity}

The COMPASS [3, 4] experiment makes use of a high energy, intense, polarised muon beam naturally polarised by the $\pi$ - decay mechanism. It uses the polarised target system of the SMC experiment, which consists of two ${ }^{6} \mathrm{LiD}$ cells, each $60 \mathrm{~cm}$ long, located along the beam one after the other in two separate RF cavities. Data are taken therefore on the two oppositely polarised target cells simultaneously.

Hereby we discuss the analysis of the data collected in year 2002 with target polarisation oriented transversely to the beam direction. This sample ( about $200 \mathrm{pb}^{-1}$ in integrated luminosity ) consists of two periods, each 5 days long, with two opposite settings in the target spin orientation. Events were selected in which a primary vertex (with identified beam and scattered muon) was found in one of the two target cells with a least one outgoing hadron. A clean separation of muon and hadron samples was achieved by cuts on the amount of material traversed in the spectrometer. In addition, the kinematic cuts $Q^{2}>1(\mathrm{GeV} / c)^{2}, W>5 \mathrm{GeV} / c^{2}$ and $0.1<y<0.9$ were applied to the data to ensure a deep-inelastic sample above the region of the nuclear resonances and within the COMPASS trigger acceptance. The upper bound on $y$ also serves to keep radiative corrections small. SSA have been looked for both the leading hadron in the event, and for all the hadrons. The leading hadron was determined as the most energetic non-muonic particle of the primary vertex having $z>0.25$, and a transverse momentum $p_{T}^{h}>0.1$ $\mathrm{GeV} / c$. When all the hadrons coming from the primary vertex were considered, the $z$ cut was lowered to 0.20 . The final data sample had an average values for $x=0.034, y=0.33$

and $Q^{2}=2.7(\mathrm{GeV} / c)^{2}$. The average value for $z$ and $p_{T}^{h}$ are 0.44 and $0.51 \mathrm{GeV} / c$ for the leading hadron analysis, 0.38 and $0.48 \mathrm{GeV} / c$ in the other case. In transverse polarisation, one can write the number of events as follows:

$$
N\left(\Phi_{C / S}\right)=\alpha\left(\Phi_{C / S}\right) \cdot N_{0}\left(1+\epsilon_{C / S} \sin \Phi_{C / S}\right)
$$


where $\epsilon$ is the amplitude of the experimental asymmetry and $\alpha$ is a function containing the apparatus acceptance. The former amplitude can be expressed as a function of the Collins and Sivers asymmetries through the expressions:

$$
\epsilon_{C}=A_{C o l l} \cdot P_{T} \cdot f \cdot D_{N N} \quad \text { or } \quad \epsilon_{S}=A_{S i v} \cdot P_{T} \cdot f
$$

where $P_{T}(\simeq 0.45)$ is the polarisation of the target, $D_{N N}$ is the spin transfer coefficient, and $f(\simeq 0.40)$ is the target dilution factor. To eliminate systematic effects due to acceptance, in each period the asymmetry $\epsilon_{C}\left(\epsilon_{S}\right)$ is fitted separately for the two target cells from the event flux with the two target orientations using the expression:

$$
\epsilon_{C / S} \sin \Phi_{C / S}=\frac{N_{h}^{\uparrow}\left(\Phi_{C / S}\right)-R \cdot N_{h}^{\downarrow}\left(\Phi_{C / S}+\pi\right)}{N_{h}^{\uparrow}\left(\Phi_{C / S}\right)+R \cdot N_{h}^{\downarrow}\left(\Phi_{C / S}+\pi\right)}
$$

where $R=N_{h, t o t}^{\uparrow} / N_{h, t o t}^{\downarrow}$ is the ratio of the total number of events in the two target polarisation orientations. The results of the asymmetries plotted against the kinematic variables $x, z$ and $p_{T}^{h}$ are shown in Fig. 1 for positive (full points) and negative (open points) hadrons, taking only the leading (top plot) hadron and for all hadrons (bottom plot).

These are the first measurements of transverse spin effects on a deuteron target: within the statistical accuracy of the data, both the Collins and Sivers asymmetries turned out to be small and compatible with zero, with a marginal indication of a Collins effect at large $z$ for both positive and negative charges.

\section{REFERENCES}

1. V. Barone, A. Drago and P. G. Ratcliffe, Phys. Rept. 359 (2002) 1.

2. J. C. Collins, Nucl. Phys. B 396 (1993) 161.

3. G. Baum et al. [COMPASS Collaboration], CERN-SPSLC-96-14.

4. A. Bressan et al. , "Recent results from COMPASS", these proceedings.

5. D. W. Sivers, Phys. Rev. D 41 (1990) 83.

6. M. Anselmino, V. Barone, A. Drago, F. Murgia arXiv:hep-ph/0209073.

7. S.J. Brodsky, D.S. Hwang, I. Schmidt, Phys. Lett. B 530 (2002) 99.

8. J. C. Collins, Phys. Lett. B 536 (2002) 43. 

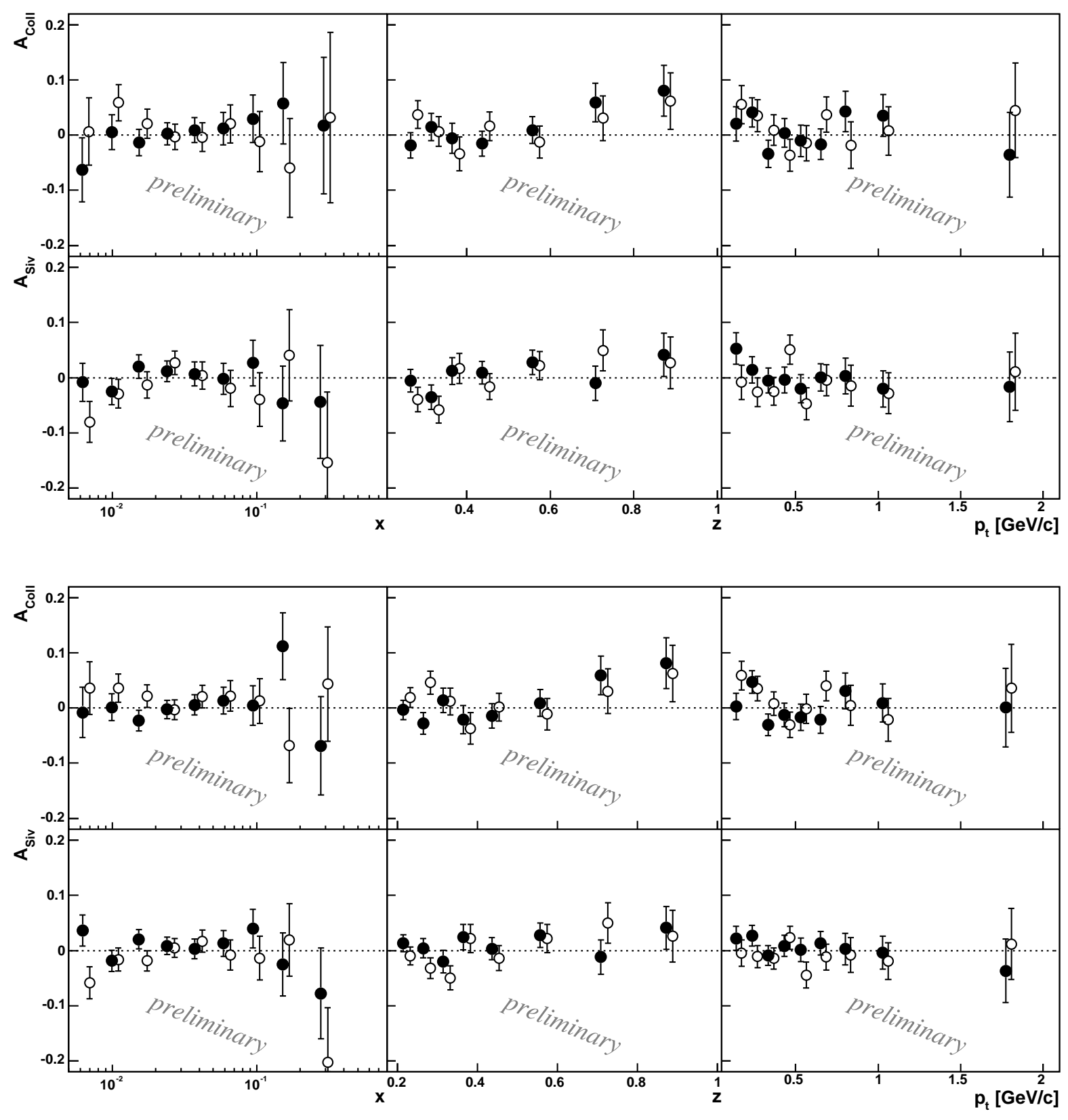

Figure 1. Collins and Sivers asymmetry for positive (full points) and negative (open points) hadrons as a function of $x, z$ and $p_{T}^{h}$. Leading hadrons analysis on top canvas, all hadrons on bottom canvas. 